\title{
Numerical Investigation of Droplet Impact on Smooth Surfaces with Different Wettability Characteristics: Implementation of a dynamic contact angle treatment in OpenFOAM
}

\author{
Vontas K. ${ }^{1,2}$., Andredaki M. ${ }^{1}$, Georgoulas A. ${ }^{1}$, Nikas K.-S. ${ }^{2}$, Marengo M. ${ }^{1}$ \\ ${ }^{1}$ Advanced Engineering Centre, School of Computing Engineering and Mathematics, \\ University of Brighton \\ ${ }^{2}$ Mechanical Engineering Department, Piraeus University of Applied Sciences \\ *Corresponding author: A.Georgoulas@brighton.ac.uk
}

\begin{abstract}
The "Direct Numerical Simulations" (DNS) of droplet impact processes is of great interest and importance for a variety of industrial applications, where laboratory experiments might be difficult, costly and time-consuming. Furthermore, in most cases after validated against experimental data, they can be utilised to further explain the experimental measurements or to extend the experimental runs by performing "virtual" numerical experiments. In such "DNS" calculations of the dynamic topology of the interface between the liquid and gas phase, the selected dynamic contact angle treatment is a key parameter for the accurate prediction of the droplet dynamics. In the present paper, droplet impact phenomena on smooth, dry surfaces are simulated using three different contact angle treatments. For this purpose, an enhanced VOF-based model, that accounts for spurious currents reduction, which has been previously implemented in OpenFOAM CFD Toolbox, is utilised and further enhanced. Apart from the already implemented constant and dynamic contact angle treatments in OpenFOAM, the dynamic contact angle model of Kistler, that considers the maximum advancing and minimum receding contact angles, is implemented in the code. The enhanced VOF model predictions are initially compared with literature available experimental data of droplets impacting on smooth surfaces with various wettability characteristics. The constant contact angle treatment of OpenFOAM as well as the Kistler's implementation show good qualitative and quantitative agreement with experimental results up to the point of maximum spreading, when the spreading is inertia dominated. However, only Kistler's model succeeds to accurately predict both the advancing and the recoiling phase of the droplet impact, for a variety of surface wettability characteristics. The dynamic contact angle treatment fails to predict almost all stages of the droplet impact. The optimum version of the model is then applied for 2 additional series of parametric numerical simulations that identify and quantify the effects of surface tension and viscosity, in the droplet impact dynamics.
\end{abstract}

\section{Keywords}

Droplet impact, dynamic contact angle treatment, VOF, OpenFOAM

\section{Introduction}

In the last 20 years, many investigations of droplet impact have taken place experimentally as well as numerically. Wettability constitutes an important controlling parameter in the dynamics of droplet impact, as it can completely alter the impact characteristics and output [1]. The "Direct Numerical Simulations" (DNS) of droplet impact processes is of great interest and importance for a variety of industrial applications, where laboratory experiments might be difficult, costly and time-consuming. Furthermore, in most cases after validated against experimental data, they can be utilised to further explain the experimental measurements or to extend the experimental runs by performing "virtual" numerical experiments. In such "DNS" calculations of the dynamic topology of the interface between the liquid and gas phase, the selected Dynamic Contact Angle (DCA) treatment is a key parameter for the accurate prediction of the droplet dynamics, since it underpins the wettability characteristics of the simulated phenomenon. The droplet impact of water on a flat, solid surface has been studied by Pasandideh-Fard et al. [2]. A numerical solution of the Navier-Stokes equations, using a modified SOLA-VOF method was utilised to model the interface deformation. Yokoi et al. [3] investigated liquid droplet impact behaviour onto a dry and flat surface numerically and compared their results with experimental data, indicating the significant role of the DCA modelling in reproducing the droplet impact behaviour. Their numerical method consists of a Coupled Level Set and VOF framework (CLSVOF), a volume/surface integrated average based multi-moment method, and a continuum surface force model. The experimental work of Antonini et al. [4] is focused in the understanding of the effect of surface wettability on impact characteristics of water drops, onto solid dry surfaces. Their results indicated the role of advancing contact angle and contact angle hysteresis as fundamental wetting parameters. They also found that, generally, if Reynolds $(\mathrm{Re})$ and Weber numbers (We) are high enough, the spreading drop can be 
subdivided into two main regions: a radially spreading lamella and an almost circular rim appearing due to capillary forces and viscosity [4]. A novel numerical implementation for the adhesion of liquid droplets impacting normally on solid dry surfaces was presented by Malgarinos et al. [5]. The benefit of this new approach, compared to most existing models, is that the DCA forming during the surface wetting process is not inserted as a boundary condition, but is derived implicitly by the induced fluid flow characteristics and the adhesion physics of the gas-liquid-solid interface, starting only from the advancing and receding equilibrium contact angles. Zhang et al. [6] investigated the phenomenon of spray impinging on in-cylinder walls, a phenomenon that has important impact on combustion processes as well as harmful emissions for internal combustion engines. Droplet impact with a numerical methodology for modelling contact line motion in a Dual-Grid Level-Set method (DGLS), on hydrophobic and super-hydrophobic surfaces was implemented by Patil et al. [7], using a quasi - DCA model based on experimental inputs. The accuracy of the partially refined DGLS method is close to that of the fine-grid based LS method, at a computation cost which is close to that of the coarse-grid based LS method. Furthermore, the DGLS method is demonstrated as an improved LS method for computational multi-fluid dynamics simulations, involving contact line motion.

In the present paper, droplet impact phenomena on smooth, dry surfaces are simulated utilising and comparing three different numerical treatments for the contact angle at the solid-liquid-gas triple contact line against existing, literature available, experimental measurements. For this purpose, an enhanced VOF-based model that accounts for spurious currents reduction, which has been previously implemented in OpenFOAM CFD Toolbox, and it was validated and applied for the case of adiabatic bubble dynamics [8], is further improved. In more detail, apart from the already implemented Constant Contact Angle (CCA) and DCA treatments in OpenFOAM, the DCA treatment of Kistler, that considers the limiting advancing and receding contact angles, is also implemented in the code. In the first part of the paper, the predictions of the three different contact angle treatments in the utilised enhanced VOF models are compared with literature reported experimental data of droplets impacting on smooth surfaces [2]. The CCA and Kistler's DCA models show good qualitative and quantitative agreement with the experimental results reported by Pasandideh-Fard et al. [2] up to the point of maximum spreading, when the spreading is inertia dominated. However, only Kistler's model succeeds to accurately predict the recoiling phase of the droplet impact. The original DCA model of OpenFOAM fails to predict almost all stages of the simulated droplet impact case. Then Kistler's treatment implementation, as it performs better, is further validated by the numerical reproduction of three additional experiments with varying wettability characteristics $[3,7]$. The proposed cases vary from hydrophilic to hydrophobic. The optimum version of the proposed numerical framework is then applied for two additional series of parametric numerical simulations (virtual experiments) that isolate, identify and quantify the effects of surface tension and viscosity, in the droplet impact dynamics. The effect of the varied parameters on the droplet spreading factors with time is identified and quantified and comparisons with the theoretical correlation by Roisman for the maximum droplet spreading factor are also conducted [9]. The proposed correlation is given by Equation 1,

$$
\beta_{\max }=0.87 R e^{1 / 5}-0.4 R e^{2 / 5} W e^{-1 / 2}
$$

\section{Numerical Method}

With the VOF approach, the transport equation for the volume fraction, $\alpha$, of the secondary (dispersed) phase is solved simultaneously with a single set of continuity and Navier-Stokes equations for the whole flow field. The corresponding volume fraction of the primary phase is simply calculated as $(1-\alpha)$. The main underlying assumptions are that the two fluids are Newtonian, incompressible, and immiscible. The governing equations can be written as:

$$
\begin{aligned}
& \nabla \cdot \vec{U}=0 \\
& \frac{\partial \rho_{b} \vec{U}}{\partial t}+\nabla \cdot\left(\rho_{b} \vec{U} \vec{U}\right)=-\nabla p+\nabla \cdot \mu_{b}\left(\nabla \vec{U}+\nabla U^{T}\right)+\rho_{b} f+F_{S} \\
& \frac{\partial a}{\partial t}+\nabla \cdot(\alpha \vec{U})-\nabla \cdot\left(\alpha(1-\alpha) U_{r}\right)=0
\end{aligned}
$$

where the bulk fluid properties are calculated as weighted averages of the individual phase properties as follows, 


$$
\begin{aligned}
& \rho_{b}=\rho \alpha+\hat{\rho}(1-\alpha) \\
& \mu_{b}=\mu \alpha+\hat{\mu}+\hat{\mu}(1-\alpha)
\end{aligned}
$$

In the VOF method, $\alpha$ is advected by the velocity field. For the case of incompressible flow, this is equivalent to volume fraction conservation, which makes the method mass conservative. Interface sharpening is very important in simulating two-phase flows of two immiscible fluids. In OpenFOAM the sharpening of the interface is achieved artificially by introducing an extra compression term in the advection equation of $\alpha$ (last term in the left-hand side of Eq. 4). $U_{r}$ is the artificial compression velocity which is calculated from the following relationship,

$$
U_{r}=n_{f} \min \left[C_{\gamma} \frac{|\varphi|}{\left|s_{f}\right|}, \max \left(\frac{|\varphi|}{s_{f}}\right)\right]
$$

Finally, the surface tension force is modelled as a volumetric force using the Continuum Surface Force (CSF) method by Brackbill et al. [10], applying the following equations:

$$
\begin{aligned}
& F_{S}=\gamma \mathrm{K}(\nabla \alpha) \\
& \mathrm{K}=\nabla \cdot\left(\frac{\nabla \tilde{\alpha}}{|\nabla \tilde{\alpha}|}\right)
\end{aligned}
$$

where $\gamma$ is the surface tension coefficient and $\mathrm{k}$ is the curvature of the interface. As mentioned in the introduction section of the present paper the utilized numerical framework constitutes an enhanced version of the original VOF-based solver of OpenFOAM [8], that suppresses numerical artefacts of the original model, known as "spurious currents". The proposed enhancement involves the calculation of the interface curvature $\mathrm{k}$ using the smoothed volume fraction values $\widetilde{\alpha}$, which are obtained from the initially calculated $\alpha$ field, smoothing it over a finite region near the interface. All other equations are using the initially calculated (non-smoothed) volume fraction values of $\alpha$. Further details on the proposed numerical modelling framework can be found in [8].

In OpenFOAM, there are two ways to predict the evolution of the contact angle between the liquid-gas interface and the solid wall boundary. The simplest approach is to assign a constant value equal to the equilibrium contact angle, and therefore neglecting the contact angle hysteresis. This is usually known as static or constant contact angle treatment. A more complicated approach involves the application of a contact angle that varies with respect to the instantaneous flow quantities. Such treatments are known as dynamic contact angle treatments (DCA). The original distribution of OpenFOAM includes both a CCA treatment as well as a DCA treatment.

For the purposes of the present investigation, after the satisfactory predictions in a similar investigation by Criscione et al. [11], the adopted, enhanced, VOF-based solver from the work of Georgoulas et al. [8], is further improved by implementing an additional DCA treatment in the solver. The proposed treatment, is known as Kistler's model [12], and it calculates the DCA, $\theta_{d}$, using the Hoffman function, $f_{\text {Hoff }}$, as follows:

$$
\theta_{d}=f_{\text {Hoff }}\left[C_{a}+f_{H o f f}^{-1}\left(\theta_{\varepsilon}\right)\right]
$$

where $\theta_{\epsilon}$ is the equilibrium contact angle. The capillary number, $C_{a}$, is calculated as $C_{a}=\frac{\mu U_{C L}}{\gamma}$ and $U_{\mathrm{CL}}$, is the spreading velocity of the contact line. $f_{\text {Hoff }}^{-1}$ is the inverse function of "Hoffman's" empirical function which is given in the following form [11].

$$
f_{\text {Hoff }}=\operatorname{acos}\left[1-2 \tanh \left(5.16\left(\frac{x}{1+1.31 x^{0.99}}\right)^{0.706}\right)\right]
$$

\section{Validation of Numerical Method}

All simulations presented in the present paper constitute $2 \mathrm{D}$ axisymmetric runs. The computational domain is a $5^{\circ}$ wedge, with $5 \mathrm{~mm}$ width and $8 \mathrm{~mm}$ height. The computational mesh consists of 1.6 million hexahedral cells $(1000 \times 1600 \times 1)$. The dimensions of the computational domain and the total number of computational cells, were 
selected after an initial sensitivity analysis and a mesh independency study, respectively. The computational domain, mesh and boundary conditions are illustrated in Figure 1. To validate the adopted, enhanced, VOF-based solver for droplet impact cases and compare the numerical predictions with the previously mentioned, contact angle treatments, four different, literature available, experiments on droplet impact are numerically reproduced. In the first case, all three contact angle models are tested, while in the rest three cases only Kistler's dynamic contact angle is used, to reproduce the proposed experimental runs. The initial conditions and the wetting characteristics of the proposed experimental runs are summarized in Table 1.

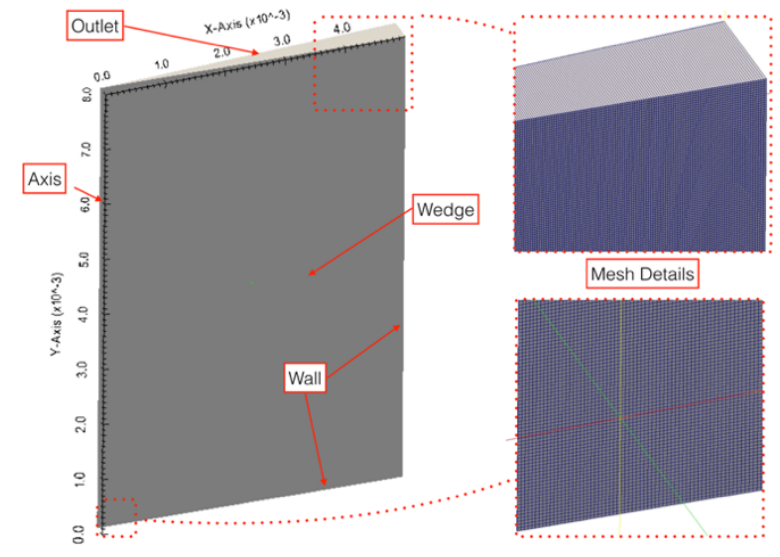

Figure 1. Computational domain, mesh and boundary conditions for numerical simulations

As it can be seen for the axisymmetric simulations of the present paper, a structured computational mesh, consisting of hexahedral and prismatic elements was used, with grid clustering towards the bottom left corner of the computational domain (centre of droplet impact). At the solid walls, a no-slip velocity boundary condition was used with a fixed flux pressure boundary condition for the pressure values and a contact angle condition, according to the selected in each case treatment, for the volume fraction values. These contact angle boundary conditions are used to correct the surface normal vector, and therefore adjust the curvature of the interface near the wall, in relation to the prescribed wettability characteristics. At the outlet, a fixed valued (atmospheric) pressure boundary condition and a zero-gradient boundary condition for the volume fraction were used, while for the velocity values a special (combined) type of boundary condition was used that applies a zero-gradient when the fluid mixture exits the computational domain and a fixed value condition to the tangential velocity component, in cases that fluid enters the domain. Further details regarding the utilised boundary conditions can be found in OpenFOAM Documentation [13].

Table 1. Initial conditions and wetting characteristics of validation experiments

\begin{tabular}{c|ccccccc}
\hline \hline & $\mathrm{D}_{0}[\mathrm{~m}]$ & $\mathrm{U}_{0}[\mathrm{~m} / \mathrm{s}]$ & $\mathrm{We}$ & $\mathrm{Re}$ & $\theta_{\mathrm{e}}\left[^{\mathrm{O}}\right]$ & $\left.\theta_{\mathrm{a}}{ }^{0}\right]$ & $\theta_{\mathrm{r}}\left[^{0}\right]$ \\
\hline \hline Experiment I [2] & 0.002 & 1 & 27 & 2000 & 90 & 110 & 40 \\
Experiment II [3] & 0.00228 & 1 & 32 & 2280 & 90 & 107 & 77 \\
Experiment III [7] & 0.0017 & 0.34 & 2.7 & 578 & 147 & 161 & 132 \\
Experiment IV [7] & 0.002 & 0.44 & 5.3 & 880 & 158 & 165 & 142
\end{tabular}

As it can be observed from Table 1, the selected validation cases constitute experiments with various impact as well as wettability characteristics. Experiments I and II constitute droplet impacts in hydrophilic surfaces, while in experiments III and IV hydrophobic and super-hydrophobic surfaces are used for the impacts, respectively. As mention previously, in the case of Experiment I all three different contact angle treatments are used to numerically reproduce the considered droplet impact. A macroscopic comparison of the numerical predictions for the droplet evolution with the corresponding experimental snapshots at the same time instances, for each treatment, is illustrated in Figure 2. For a more quantitative comparison, the contact diameter of the droplet with the solid surface with respect to time is plotted for each of the three numerical simulations as well as for the experimental measurements, in the diagram of Figure 3. 


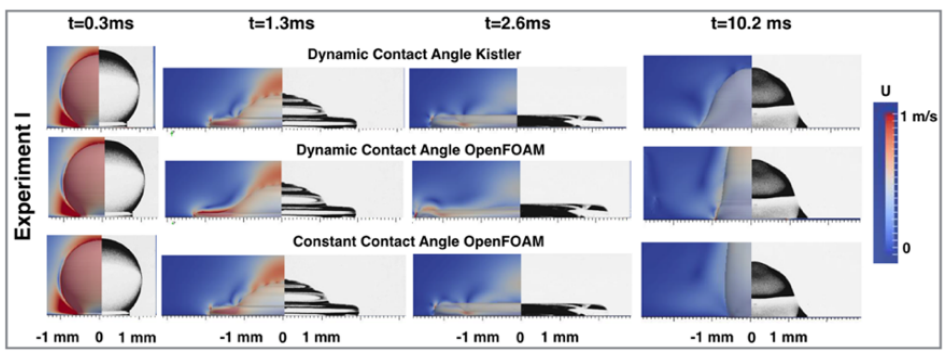

Figure 2. Droplet evolution with time - Comparison of numerical predictions for all three contact angle treatments (present investigation) with corresponding experimental snapshots from [2]. The velocity magnitude field in a central vertical section of the droplet, is also shown in the numerical snapshots.

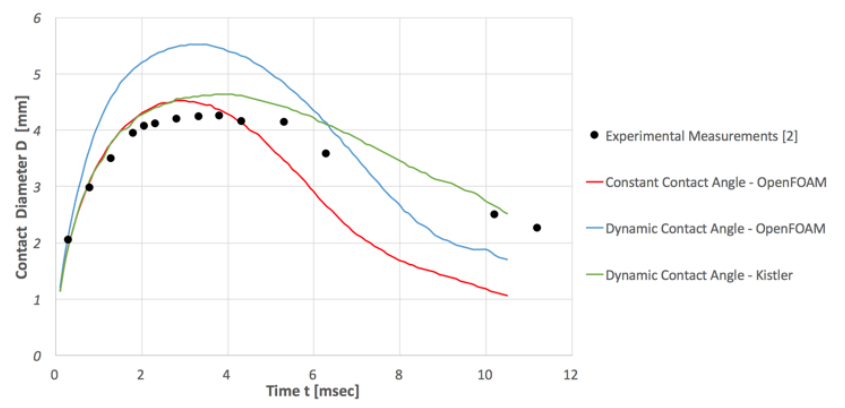

Figure 3. Contact diameter with time - Comparison of numerical predictions (present investigation) for all three contact angle treatments with corresponding experimental data from [2].

After examining Figures 2 and 3, it is obvious that both the CCA and DCA treatments implemented in the original distribution of OpenFOAM fail to predict the experimental data, while Kistler's DCA treatment follows the experimental points well, both before and after the maximum spreading. However, in order to further check the validity of the Kistler's DCA treatment for cases with varying wettability characteristics, three additional, literature available experimental runs that are reported in [3] and [7], are numerically reproduced. In more detail, another hydrophilic (Experiment II), a hydrophobic (Experiment III) and a super-hydrophobic case (Experiment IV) are selected for this purpose. A macroscopic comparison of the numerical predictions for the droplet evolution with the corresponding experimental snapshots, is illustrated in Figure 4. For a more quantitative comparison, the contact diameter of the droplet with the solid surface with respect to time, is plotted for each case in the diagrams of Figure 5.

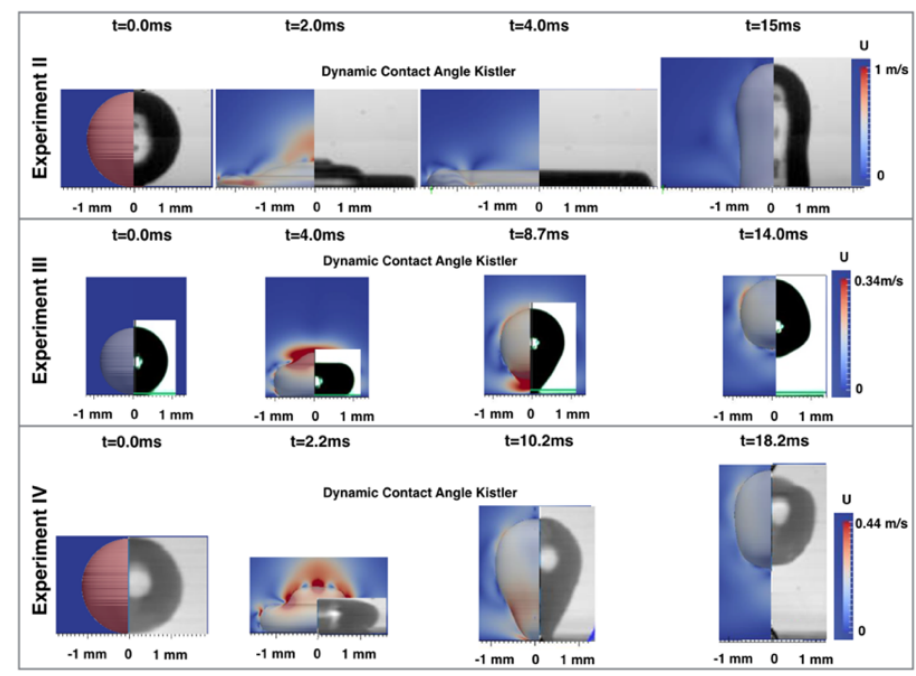

Figure 4. Droplet evolution with time - Comparison of numerical predictions (present investigation) using Kistler's dynamic contact angle treatment with corresponding experimental snapshots reported in [3] (top) and [7] (middle) and (bottom). The velocity magnitude field in a central vertical section of the droplet, is also shown in the numerical snapshots. 


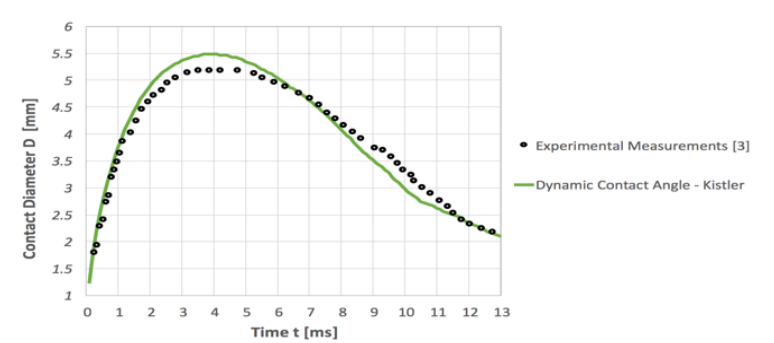

(a)

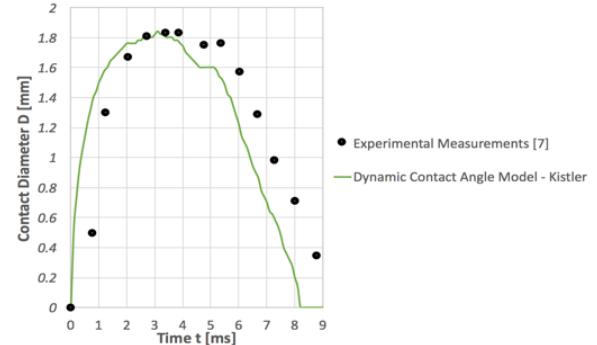

(b)

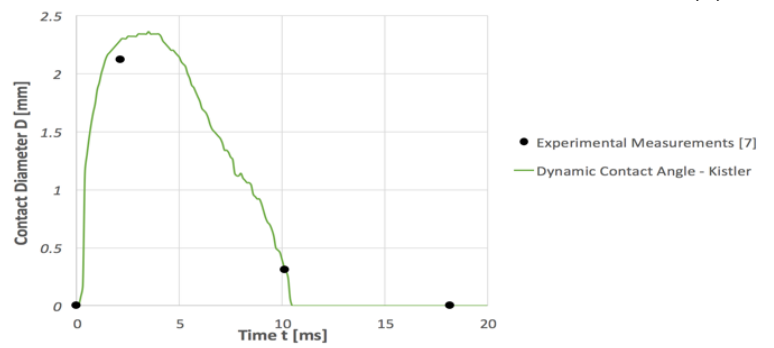

(c)

Figure 5. Contact diameter with respect to time - Comparison of numerical predictions (present investigation) using Kistler's dynamic contact angle treatment with corresponding experimental snapshots reported in [3] (a) and [7] (b) and (c).

As it can be observed, Kistler's DCA treatment successfully predicts the spatial and temporal evolution of the droplet in all stages of the considered impacts. Therefore, it can be concluded that the utilised enhanced VOF framework in conjunction with the implemented DCA treatment of Kistler, can safely be applied for the investigation of droplet impacts through "virtual" numerical experiments, as it successfully predicts the droplet impact stages in hydrophilic, hydrophobic and super-hydrophobic cases.

\section{Parametric Analysis - Effect of fluid viscosity and surface tension}

In the present section of the paper, the optimum version of the VOF-based numerical framework that is presented and validated against experimental data in the previous sections, is further applied for the conduction of two additional series of parametric numerical simulations. The proposed numerical experiments mainly aim to identify and quantify the effects of viscosity and surface tension on the spatial and temporal evolution of the droplets after their impact on a hydrophilic surface. Furthermore, the validity of a widely used theoretical correlation by Roisman [9] (Equation 1) for the case of "virtual" fluids is assessed. For this purpose, the validation case reproducing Experiment II is selected as the base case for the proposed parametric numerical investigations. In the first parametric investigation 5 additional simulations are conducted "virtually" varying only the liquid viscosity, by factors of $0.5,1.5,2.0,3.0$ and 3.5, while keeping all the other properties and impact characteristics constant and equal to the base case. In the second parametric investigation, again 5 additional simulations are conducted virtually varying only the surface tension coefficient, by factors of $0.5,1.5,2.0,3.0$ and 3.5 , while keeping all the other properties and impact characteristics constant and equal to the base case. The evolution of the spreading factor $\beta$ with dimensionless time $t^{*}$ for each case is plotted. The spreading factor is calculated as the contact diameter over the initial droplet diameter $\frac{D}{D_{0}}$ while the dimensionless time $t^{*}$ is calculated as $t \frac{U_{0}}{D_{0}}$. The effects of the variation of viscosity and surface tension on the spreading factor $\beta$ with respect to dimensionless time $t^{*}$ are given in Figures 6(a) and 6(b). In each case the corresponding maximum spreading factor from Equation 1 (Roisman correlation [9]) is plotted for comparison purposes. 


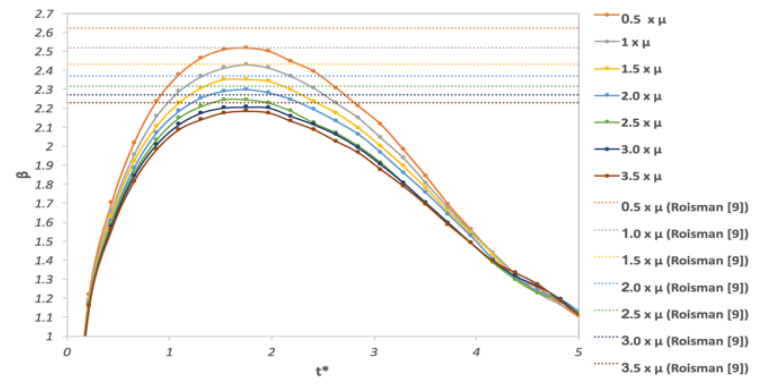

(a)

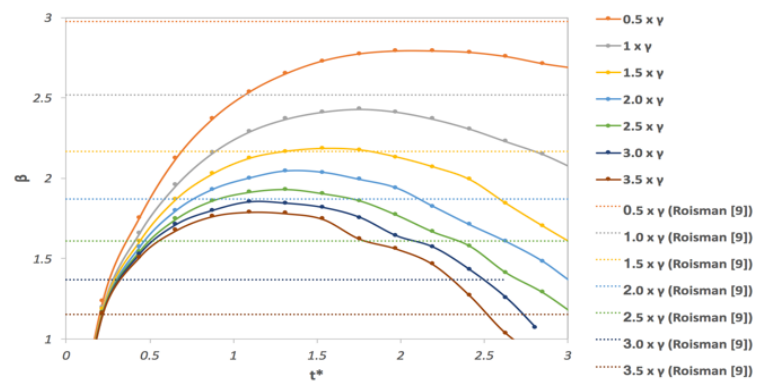

(b)

Figure 6. (a) Spreading factor vs dimensionless time - effect of viscosity variation (b) spreading factor vs dimensionless time effect of surface tension variation. Dotted lines correspond to the maximum spreading factor as predicted using the theoretical correlation of Roisman [9] (Equation 1).

As it can be observed from Figure 6 (a), as viscosity increases both the spreading as well as the recoiling stages of the droplet are dumped. It is characteristic however that the proposed effect, is mor intense around the maximum spreading stage of the drople,t while it appears to be negligible during the initial spreading and the later recoiling stages. It can also be seen that the theoretical correlation of Roisman, in each case slightly over-predicts the resulting maximum spreading factors from the numerical simulations, by $2 \%$ up to $4 \%$. Examining Figure 2 (b), it can be observed that as surface tension increases, the maximum spreading is significantly reduced and the recoiling stage is strengthened. The proposed effect is quite evident from the first stages of the spreading and it progressively increases towards the last stages of the recoiling phase. It is characteristic that a variation of the surface tension coefficient by a factor of just 0.5 can significantly alter the post-impact dynamics of the droplet. As for the comparison with the theoretical correlation of Roisman an increasing deviation from the numerically predicted maximum spreading factor is observed with the corresponding increase of the surface tension. The deviation between the theoretical and numerical maximum spreading factors in this case varies from $0.85 \%$ up to $55 \%$.

The results from the present parametric investigation illustrate that the correlation proposed by Roisman [9] can be safely used for the prediction of the maximum spreading of viscous fluids with viscosities up to 3.5 times higher than water, but it significantly fails to predict the maximum spreading for fluids with surface tension more than two times the surface tension of water, such as metal fluids.

\title{
Conclusions
}

In the present paper, Kistler's DCA model has been implemented in a previously improved version of the VOFbased solver of OpenFOAM. The performance of the proposed contact angle treatment is compared with the existing contact angle treatments of OpenFOAM's original distribution, through comparison with literature reported experimental measurements on water droplets impacting on hydrophilic surfaces. To further test the revealed accuracy of the proposed DCA implementation, three additional experimental runs are numerically reproduced; a second hydrophilic case as well as a hydrophobic and a super-hydrophobic case. In all cases, Kistler's model implementation in the utilised, enhanced VOF-based solver, successfully predicts the spreading, recoiling and rebounding stages of the droplet impact. Further application of the numerical model for the conduction of two additional series of parametric numerical experiments identifies and quantifies the effects of viscosity and surface tension variation in the post-impact stages of the droplet evolution. Comparison of the numerical predictions with a widely accepted theoretical correlation [9], indicate that the proposed correlation can be safely applied to predict the maximum spreading of fluids with higher viscosity than water but not for fluids with more than two times the surface tension of water. Finally, from the overall presentation and analysis of the results it is obvious that the proposed enhanced VOF framework can be safely used to further examine the effects of a variety of important controlling parameters to the post-impact characteristics of droplets impinging on solid surfaces with various wetting characteristics, ranging from hydrophilic to super-hydrophobic cases.

\author{
Nomenclature \\ $\beta_{\max } \quad$ maximum spreading factor [-] (contact droplet diameter / initial droplet diameter) \\ Re Reynolds Number [-] \\ We Weber Number [-] \\ $\vec{U} \quad$ velocity vector $\left[\mathrm{ms}^{-1}\right]$ \\ $\rho_{b} f \quad$ volumetric representation of the gravitational force $\left[\mathrm{kg} / \mathrm{m}^{2} \mathrm{~s}^{2}\right]$ \\ $p \quad$ pressure $[\mathrm{Pa}]$
}




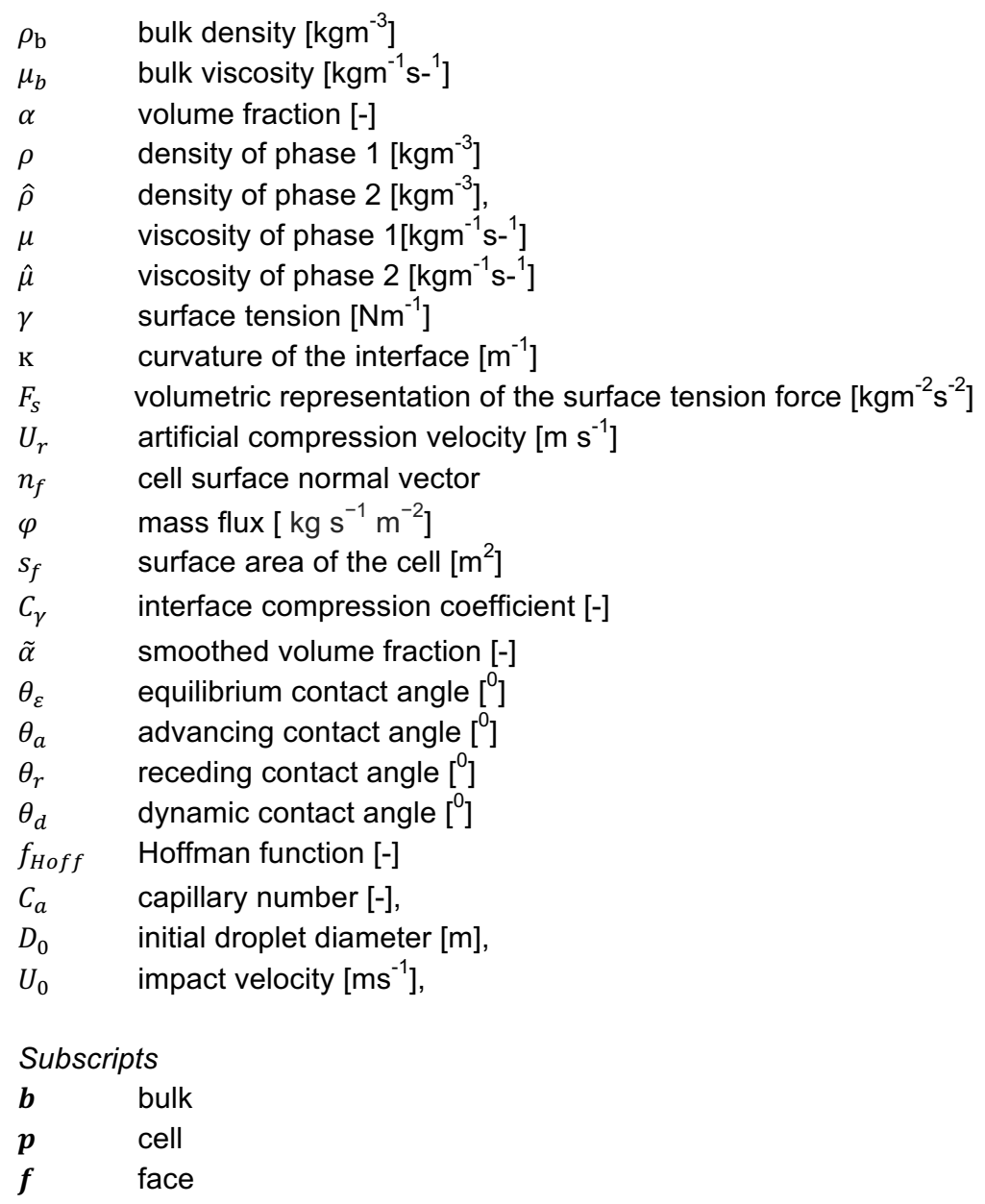

\section{References}

[1] Marengo, M., Antonini, C., Roisman, I. V., and Tropea, C., 2011, "Drop Collisions with Simple and Complex Surfaces," Curr. Opin. Colloid Interface Sci., 16(4), pp. 292-302.

[2] Pasandideh-Fard, M., Y. M. Qiao, Y. M., Chandra, S., and Mostaghimi, J., 1996, "Capillary Effects during Droplet Impact on a Solid Surface," Phys. Fluids, 8(3).

[3] Yokoi, K., Vadillo, D., Hinch, J., and Hutchings, I., 2009, "Numerical Studies of the Influence of the Dynamic Contact Angle on a Droplet Impacting on a Dry surfaceNo Title," Phys. Fluids, 21(7), p. 72102.

[4] Antonini, C., Amirfazli, A., and Marengo, M., 2012, "Drop Impact and Wettability: From Hydrophilic to Superhydrophobic Surfaces," Phys. fluids, 24(10), p. 102104.

[5] Malgarinos, I., Nikolopoulos, N., Marengo, M., Antonini, C., and Gavaises, M., 2014, "VOF Simulations of the Contact Angle Dynamics during the Drop Spreading: Standard Models and a New Wetting Force Model," Adv. Colloid Interface Sci., 212, pp. 1-20.

[6] Zhang, Z., Liu, H., Zhang, F., and Yao, M., 2016, "Numerical Study of Spray Micro-Droplet Impinging on Dry/wet Wall," Appl. Therm. Eng., 95, pp. 1-9.

[7] Patil, N. D., Gada, V. H., Sharma, A., and Bhardwaj, R., 2016, "On Dual-Grid Level-Set Method for Contact Line Modeling during Impact of a Droplet on Hydrophobic and Superhydrophobic Surfaces," Int. J. Multiph. Flow, 81, pp. 54-66.

[8] Georgoulas, A., Koukouvinis, P., Gavaises, M., and Marengo, M., 2015, "Numerical Investigation of Quasi-Static Bubble Growth and Detachment from Submerged Orifices in Isothermal Liquid Pools: The Effect of Varying Fluid Properties and Gravity Levels," Int. J. Multiph. Flow, 74, pp. 59-78.

[9] Roisman, I., 2009, "Inertia Dominated Drop Collisions. II. An Analytical Solution of the Navier-Stokes Equations for a Spreading Viscous Film," Phys. fluids, 21(52104).

[10] Brackbill, J. ., Kothe, D. ., and Zemach, C., 1992, "A Continuum Method for Modeling Surface Tension," J. Comput. Phys., 100(2), pp. 335-354.

[11] Criscione, A., Rohrig, R., Opfer, L., Roisman, I., and Jakirlic, S., 2011, "Numerical Investigation of Impacting Water Drops in Air Cross-Flow," ILASS-Europe 2011, 24th European Conference on Liquid Atomization and Spray Systems, Estoril, Portugal.

[12] Kistler, S., 1993, "Hydrodynamics of Wetting," Wettability, 6(Marcel Dekker, New York), pp. 311--430.

[13] CFD_Direct, 2013, "The OpenSource CFD Toolbox, User Guide." 